\title{
LOS ITINERARIOS FÍSICO-DEPORTIVOS DE LAS PERSONAS MAYORES Y SUS FACTORES DETERMINANTES
}

\author{
Marcos Gonçalves Maciel \\ Universidade do Estado de Minas Gerais (Brasil) \\ María Jesús Monteagudo Sánchez \\ Universidad de Deusto
}

\begin{abstract}
RESUMEN: Esta investigación cualitativa busca conocer los factores, presentes y pasados, responsables de la adherencia físico-deportiva de un grupo de personas de 60 años o más, residentes en Bilbao (España). Se parte de una muestra intencional de 18 personas (10, físicamente activas y ocho, inactivas durante el estudio). El análisis de sus historias físico-deportivas, reconstruidas mediante entrevistas semiestructuradas en profundidad, ha permitido identificar seis itinerarios físico-deportivos que evidencian el impacto de algunos factores en la evolución de la práctica físico-deportiva a lo largo de la vida. Entre ellos, destacan factores implicados en la decisión actual de las personas mayores de estar o no físicamente activas (salud, apoyo familiar, centros polideportivos de proximidad) y otros, asociados a sus historias físico-deportiva (falta de tiempo y oportunidades, ética del trabajo, ausencia de prácticas previas o experiencias negativas). Se confirma el influjo del contexto sociohistórico asociado a etapas pasadas de la vida en el ocio físicamente activo o inactivo durante la tercera edad. La apuesta por la adherencia físico-deportiva de las personas mayores implica, no solo intervenir en esta fase tardía, sino también en etapas anteriores, propiciando las condiciones idóneas para hacer de este ámbito una oportunidad para mejorar la salud y bienestar de este colectivo.
\end{abstract}

PALABRAS CLAVE: itinerarios de ocio, prácticas físico-deportivas, factores determinantes, personas mayores, ciclo vital.

\section{ITINERARY OF PHYSICAL AND SPORTS ACTIVITIES OF OLDER PEOPLE AND ITS CORRELATES}

ABSTRACT: This qualitative research seeks to know the factors, present and past, responsible for the physical-sports adherence of a group of people aged 60 years or more, residing in Bilbao (Spain). It starts with an intentional sample of 18 people (10, physically active and eight, inactive during the study). The analysis 
of their physical-sports histories, reconstructed through in-depth semi-structured interviews, has allowed the identification of six physical-sports itineraries that show the impact of some factors on the evolution of physical-sports practice throughout life. Among them, factors involved in the current decision of the elderly to be physically active or not (health, family support, local sports centers) and others, associated with their physical-sports histories (lack of time and opportunities, ethics of the work, absence of previous practices or negative experiences). The influence of the socio-historical context associated with past stages of life in physically active or inactive leisure during old age is confirmed. The commitment to physical-sports adherence of the elderly implies, not only intervening in this late phase, but also in earlier stages, providing the ideal conditions to make this area an opportunity to improve the health and well-being of this group.

KEYWORDS: Leisure itineraries, physical-sports practices, correlates, elderly people, life cycle.

Recibido: $21 / 11 / 2020$

Aceptado: 10/01/2021

Correspondencia: María Jesús Monteagudo Sánchez, Instituto de Estudios de Ocio, Universidad de Deusto, Avenida de las Universidades, 24, 48007 Bilbao. Email: mjmonte@deusto.es

\section{INTRODUCCIÓN}

El envejecimiento de la población es una realidad mundial que causa impactos en diversos sectores (WHO, 2004, 2015), entre ellos, economía, empleo, educación, urbanización, salud. En España, este proceso se inició más tarde que en el resto de países del área meridional europea, pero se consolidó a un ritmo más acelerado (IMSERSO, 2017). Las previsiones indican que la población de 65 años y más seguirá aumentando en España hasta el año 2050 y en 2060, representará más de un tercio del total de la población española (35,6 \%) (IMSERSO, 2017).

Ante este hecho, gobiernos, organismos internacionales y universidades investigan los factores que interfieren en los aspectos biopsicosociales de las personas mayores, con el propósito de mejorar su calidad de vida, manteniendo la dignidad y el protagonismo social. Una de las acciones más extendidas para ello es la promoción de actividades físico-deportivas, entendidas como prácticas de ocio. Numerosos estudios demuestran su impacto positivo en la salud a diferentes niveles, que van desde la mejora de la autoestima, la función cognitiva, la reducción del riesgo de caídas y dolores osteoarticulares, hasta la mitigación de situaciones de soledad o estados de depresión (Aparicio, Cabonell y Delgado, 2010), cada vez más comunes en esta etapa. Pese a que estos y otros beneficios biopsicosociales son ampliamente conocidos, la prevalencia de situaciones de sedentarismo entre la población mayor sigue siendo alta, consolidándose como tendencia que preocupa a Estados y organismos internacionales como la Organización Mundial de la Salud (Guthold, Stevens, Riley y Bull, 2018; Picorelli, Felicio, Pereira, Pereira y Sherrington, 2014; WHO, 2015). Estos 
mismos estudios ratifican que el sedentarismo afecta en mayor medida a las mujeres, evidenciándose el género como un factor a tener en cuenta (Aitchison, 2003; Martín, Moscoso, Martínez del Castillo y Ferro, 2009). Entre otras razones, por las múltiples barreras para la práctica físico-deportiva que experimentan asociadas a los roles sociales tradicionalmente desempeñados (tareas domésticas, crianza de hijos e hijas y de otros familiares) y a los que se suma, muchas veces, el trabajo fuera de casa (Brown, Brown, Miller y Hansen, 2001, Currie, 2004).

La mayoría de los estudios analizan el problema desde una mirada sincrónica (Castañeda, Campos y Garrido, 2011; Lopes, Longo, Peres, Boing y Arruda, 2010); de ahí el interés de investigaciones que abordan el reto de la adherencia físico-deportiva en personas mayores con un enfoque retrospectivo y diacrónico, explorando las experiencias físico-deportivas habidas en etapas anteriores, así como los factores promotores o inhibidores de las mismas (Langley y Knight, 1999).

Desde los Estudios de Ocio, este enfoque retrospectivo de la trayectoria vital toma forma en el concepto itinierario de ocio, entendido como el conjunto de experiencias de ocio que la persona ha disfrutado en su historia de vida. En el caso del ocio físicodeportivo, un itinerario se "inicia en el instante en que la persona toma contacto con este ámbito; se desarrolla durante un tiempo más o menos amplio en el que se dedica a la práctica de una o varias actividades de ocio físico-deportivo y finaliza cuando se abandona toda relación con dichas prácticas" (Monteagudo y Cuenca, 2012:108) ${ }^{1}$. Habitualmente, un itinerario transcurre por tres fases: inicio, momento en el que la persona comienza su relación con la práctica físico-deportiva; desarrollo, cuando la persona protagonista decide continuar o abandonar sus prácticas; y, en caso de continuar, la fase de resolución, en la que la persona pone fin a su participación mediante el abandono definitivo de la práctica.

La literatura científica asume el hecho físico-deportivo como resultado de un complejo entramado de factores internos (personales) y externos (sociales) (Nahas, Goldfine y Collins, 2003) que denomina factores determinantes. Ambos tipos de factores se caracterizan por su capacidad para influir en las prácticas físico-deportivas y su evolución en direcciones opuestas, como facilitadores, promoviendo la adherencia y reduciendo las conductas sedentarias, o inhibidores, actuando como barreras para un ocio físicamente activo. De ahí que su estudio resulte fundamental. El análisis del itinerario físico-deportivo permite identificar dichos factores determinantes implicados en la evolución del ocio físico-deportivo a lo largo de la vida y detectar tendencias o patrones en la práctica físico-deportiva, esenciales para prevenir futuras conductas sedentarias durante la tercera edad.

Las preguntas a las que esta investigación pretende dar respuesta son:

1. ¿Cómo son las historias físico-deportivas de las personas mayores actualmente activas e inactivas? ¿En qué se diferencian? ¿Han realizado las personas mayores estudiadas alguna práctica físico-deportiva en etapas anteriores? ¿Cuándo las iniciaron? ¿Cómo han evolucionado? ¿Qué actividades realizan actualmente?

2. ¿Qué factores (facilitadores y barreras) han incidido en sus estilos de ocio físicamente activos o inactivos actuales?

1. Definición adaptada del concepto de itinerario deportivo de Puig y Masnou (1995: 371). 
Dar respuesta a estas cuestiones es clave para alcanzar el objetivo principal de este estudio, identificar los factores pasados y presentes que contribuyen o dificultan la práctica físico-deportiva durante la tercera edad. Para ello, se reconstruyen y analizan los itinerarios físico-deportivos de este colectivo y su incidencia en su situación actual, como personas físicamente activas o inactivas.

Esta investigación se desarrolla en Bilbao (Euskadi), la ciudad vasca que presenta el mayor porcentaje de personas de 65 años o más de toda la Comunidad Autónoma (24\%, aproximadamente 82.428 habitantes) (Eustat, 2019). Conviene subrayar que la población de personas de 65 años o más alcanza en Euskadi un 22,3\%, 3 puntos porcentuales por encima de la media estatal $(19,3 \%)$ y sólo por detrás de Asturias (25,7\%), Castillla-León (25,4\%) y Galicia (25,2\%) (Pérez Díaz, Abellán García, Aceituno Nieto y Ramiro Fariñas, 2020).

\section{Método}

Adoptar el concepto de itinerario de ocio como marco de reflexión para investigar la adherencia físico-deportiva de las personas mayores requiere un diseño metodológico adecuado, capaz de interpretar los procesos narrativos mediante los que las personas integran sus experiencias físico-deportivas en sus historias vitales y el contexto sociohistórico que las acoge. De ahí que se haya optado por la investigación cualitativa, descriptiva y exploratoria y por la entrevista semiestructurada en profundidad como alternativas idóneas para este tipo de estudios (Lakatos y Marconi, 2003).

\section{Participantes}

El estudio parte de una muestra intencional, con dos perfiles: por un lado, personas de 60 años o más, residentes en Bilbao, físicamente activas; y por otro, un colectivo de idénticas características, pero físicamente inactivo. Los requisitos de participación de sendos colectivos eran: 1. tener 60 años o más, 2. residir en Bilbao, 3. estar en pleno uso de sus facultades. En el caso de las personas mayores físicamente activas, debían, además, practicar en el momento del estudio, al menos, una actividad físico-deportiva desde un tiempo mínimo de 3 meses, periodo requerido para la creación de un hábito que favorezca el mantenimiento de la actividad (Weinberg y Gould, 2014). Por el contrario, formar parte del grupo físicamente inactivo implicaba no practicar ninguna actividad físico-deportiva en el momento del estudio y no experimentar ninguna discapacidad permanente que imposibilitase la práctica físicodeportiva

La identificación de la muestra se lleva a cabo con el apoyo y consentimiento de dos órganos del Ayuntamiento de Bilbao, el Instituto Municipal de Deportes (Bilbao Kirolak), responsable de administrar los complejos polideportivos de la ciudad, en el caso de las personas físicamente activas y el Área de Acción Social, esencial para acceder a los Hogares de Jubilados², en el caso de las inactivas.

Para determinar el número suficiente de personas participantes se adopta la técni-

2. Centros públicos, cuyo objetivo es el fomento de la calidad de vida de las personas mayores mediante la oferta de actividades de ocio, creativas y participativas. 
ca de saturacion de datos (Clark y Creswell, 2008). La muestra definitiva está formada por 18 personas, 10 mujeres y ocho hombres. La edad promedio es de 70,5 años (año de nacimiento, 1948). Por rango de edad, la persona más joven tiene 62 años (nacida en 1956) y la más mayor, 82 (nacida en 1936). Un total de 10 personas están físicamente activas en el momento del estudio y ocho, inactivas.

La tabla 1 presenta los datos sociodemográficos de la muestra, según los perfiles indicados: personas físicamente activas e inactivas.

\section{INSTRUMENTO}

Atendiendo a los objetivos y tipo de muestra, se opta por la entrevista semiestructurada en profundidad para la recogida de datos. Se elabora un guión preliminar, organizado en dos apartados temáticos: 1. Datos sociodemográficos y económicos; 2. Prácticas físico-deportivas realizadas a lo largo de la vida. En este segundo apartado, se pretenden conocer las actividades físico-deportivas practicadas en periodos previos a este estudio y las que se realizan actualmente. Igualmente, mediante preguntas abiertas, se busca identificar los factores determinantes (barreras y facilitadores) de estas prácticas en cada etapa. Con una finalidad claramente operativa, las fases de la vida se asocian a categorías o franjas de edad del siguiente modo: infancia (hasta 12 años), adolescencia (13-19 años), edad adulta (20-59 años), considerando la edad adulta temprana entre 20 y 39 años y la tardía, de los 40 a 59 años y tercera edad (60 años y más).

\section{Procedimiento y análisis de datos}

Antes de la administración de la entrevista, se realiza un estudio piloto para evaluar los procedimientos metodológicos y la idoneidad del guión de entrevista. Las entrevistas se realizan "face to face" en los polideportivos designados por Bilbao Kirolak, en el caso de las personas mayores físicamente activas y, en los Hogares de Jubilados autorizados, en lo que respecta a las personas mayores físicamente inactivas. Las entrevistas se realizan en octubre de 2018. Fueron grabadas en audio y, después, transcritas para su análisis.

La interpretación de datos se realiza mediante el análisis de la historia oral (Meihy, 2006). Según esta autora, la percepción del pasado hace posible reconstruir el proceso histórico, aún inacabado e inferir ciertas líneas de futuro. Además, esta propuesta es especialmente útil para investigar las experiencias pasadas que se recuerdan, eventos, hechos personales y sociales que fueron relevantes para la persona, ayudando a reconstruir el contexto sociocultural de cada cual.

Concretamente, el análisis de la información recabada se organiza en torno a tres fases. En primer lugar, se transcriben las entrevistas y se identifican las cuestiones clave de las historias de ocio, así como los puntos críticos emergentes sobre los temas estudiados (evolución de las prácticas y factores facilitadores y/o barreras de la práctica físico deportiva a lo largo del ciclo vital). Se procede a la redacción de textos provisionales, reinterpretando las historias a la luz del marco teórico planteado y las narrativas compartidas por las personas entrevistadas. En segundo lugar, se inicia la identificación de categorías, atendiendo a las preguntas del estudio y a las cuestiones críticas de- 
MARCOS GONÇALVES MACIEL - MARÍA JESÚS MONTEAGUDO SÁNCHEZ

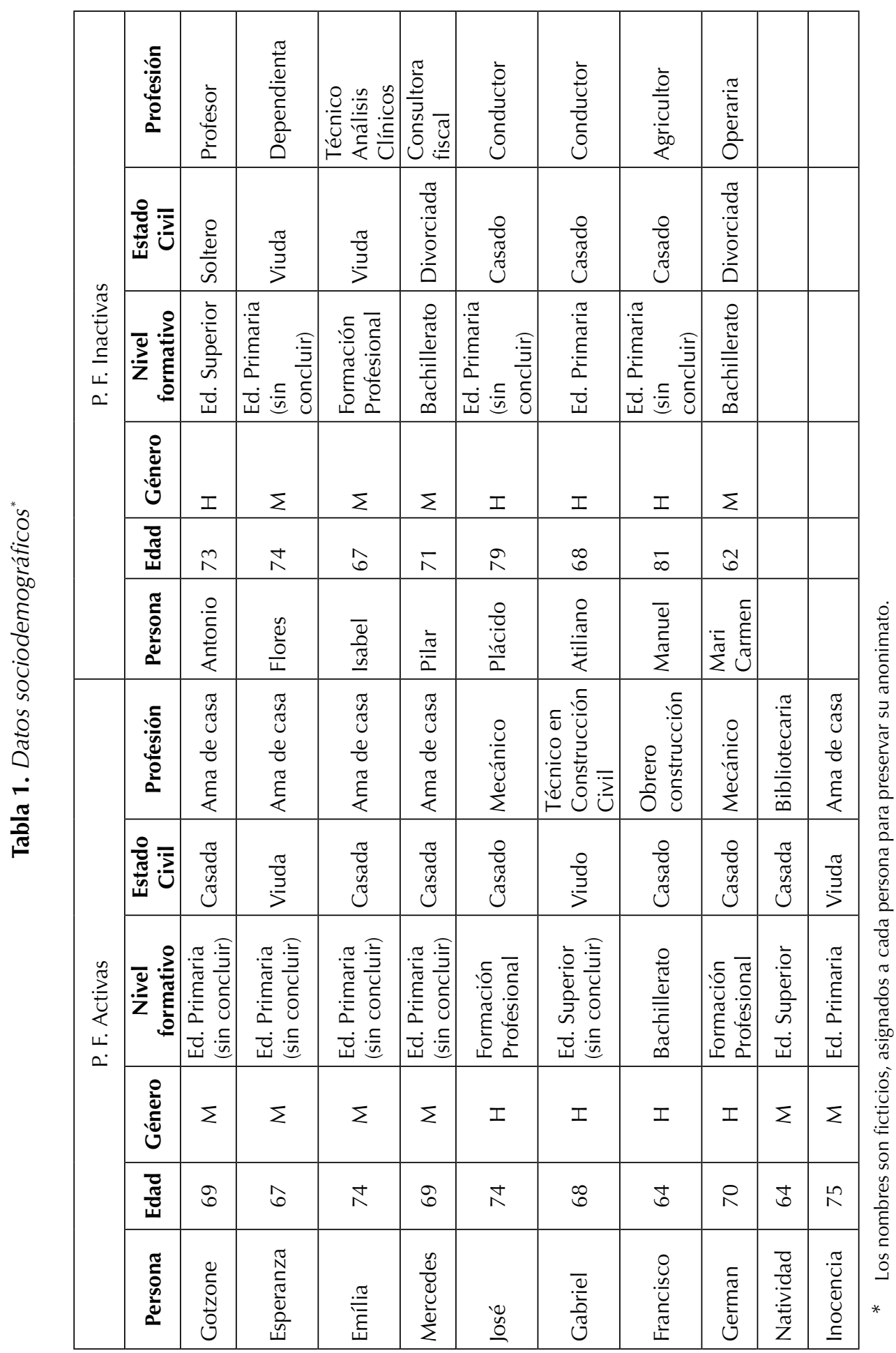


tectadas, asociadas ahora con las categorías temáticas generadas. Es el momento de identificación de conexiones temáticas entre los casos y de la detección de cuestiones particulares, que pueden arrojar luz sobre el objeto de estudio. En tercer lugar, se inicia la fase de feedback y visto bueno de las narrativas individuales y las interpretaciones realizadas por parte de las personas entrevistadas. A partir de este momento, se procede a la redacción del informe definitivo, fruto del trabajo elaborado en fases anteriores.

Con el fin de controlar posibles sesgos que amenazan la validez de la investigación cualitativa, se han tomado las siguientes medidas:

1. Considerar los límites del lenguaje para captar la complejidad y la profundidad de los significados experimentados y atribuidos a las experiencias. Para evitar interpretaciones incorrectas, se realizaron varios procesos de feedback, contrastando con cada persona los temas y cuestiones principales surgidos en el análisis de su entrevista. Se daba por buena la interpretación, cuando los resultados eran reconocidos por las personas implicadas como fiales a lo que sentían y pensaban.

2. Analizar la misma información por diferentes investigadores, com tradiciones disciplinares e intereses diversos. Así, fue posible, en primer lugar, contrastar puntos de vista distintos para analizar la información, favoreciendo una visión más rica y compleja de cada caso y, en segundo lugar, garantizar que los elementos centrales y más destacables de cada caso, eran los mismos para todos los investigadores e investigadoras.

\section{Resultados}

Los resultados se presentan organizados en dos apartados. El primero presenta cada etapa del ciclo vital (infancia, adolescencia y juventud, edad adulta y tercera edad), en clave físico-deportiva, atendiendo a las oportunidades y dificultades experimentadas para la práctica; el segundo, reconstruye las historias de vida físicodeportiva de las personas participantes para identificar y clasificar los patrones de evolución de sus prácticas en distintos itinerarios físico-deportivos.

\section{La práctica físico-deportiva a lo largo de la vida}

Para conocer cómo fue la relación de cada persona con la práctica físico-deportivo en etapas anteriores de la vida, se plantea la pregunta: "Describa qué actividades físicas-deportivas ha realizado en su tiempo libre a lo largo de su vida, desde la infancia hasta el momento actual". Las respuestas se agrupan por categorías de similitud para cada rango de edad y estilo de vida.

\section{Infancia (hasta 12 años)}

Para el análisis de este periodo, se toma como referencia el "año de nacimiento promedio" de las y los participantes, 1948; por tanto, la infancia de este colectivo tuvo lugar durante la década de 1950.

En esta etapa, las únicas experiencias físico-deportivas de este colectivo fueron en forma de juegos, en la escuela, durante su tiempo libre. Practicamente, ninguno de 
los participantes realizó prácticas físico-deportivas organizadas, más allá de las clases de Educación Física que algunos recibieron. Disfrutaron del juego por su cuenta, en soledad o con sus iguales, sin la presencia de personas adultas y, principalmente, en la calle. Algunas reflexiones de las personas protagonistas así lo confirman:

"Haciendo ejercicio como tal, no lo recuerdo. Después del colegio nuestros padres iban a la plaza y nos dejaban correr y jugar. Patinábamos, saltábamos a la cuerda, jugamos con la pelota." (Natividad, 64).

"Después de clase íbamos a casa. Jugábamos con amigos en frente de la casa y nada más." (Pilar, 71).

"Jugué al fútbol, nada más. No había otras cosas. Jugamos en las calles y en las plazas. Reuníamos a nuestros colegas y jugábamos." (Plácido, 79).

"No tuve mucho tiempo para el ocio. En la escuela, durante el recreo, se jugaba fútbol" (Germán, 70).

La Educación Física tampoco era una realidad habitual en las escuelas de esa época, por lo que sólo fue una oportunidad para el juego, el ejercicio físico y el movimiento en el caso de algunas personas.

"En las escuelas no había deportes, solo leía un poco y lanzaba una pelota en el frontón. No tenía clases de educación física... No había un sistema para promover estas clases." (Atiliano, 68).

"No recuerdo bien, pero creo que en las clases de educación física hacíamos algunos juegos y actividades. Fuera de clase, no hice nada." (Mari Carmen, 62).

\section{Adolescencia y Juventud (13-19 años)}

Debido a la realidad social de la época (situación de posguerra), a medida que los niños y niñas crecían, se veían obligados a trabajar, colaborar en las tareas del hogar y en las faenas del campo. Algunos relatos evidencian que la adolescencia era una fuerza de trabajo imprescindible para la economía familiar, quedando el derecho a la educación y, por supuesto, al ocio, relegados a un segundo plano.

"No tuve tiempo para eso. Vivíamos en el campo en Andalucía. Mi padre, cuando no nos encargábamos de criar cerdos, nos enviaba a cargar leña para hacer fuego, hacer comida y limpiar los establos de animales. De actividad física, nada. Hoy aún necesito continuar trabajando y no tengo tiempo para hacer ejercicio" (Flores, 76).

"Soy de un pueblo asturiano y teníamos vacas que cuidar. En la adolescencia, trabajaba y estudiaba. Me quedaba muy poco tiempo." (Germán, 70).

"Cuando éramos adolescentes comenzábamos a trabajar temprano y no teníamos tiempo para hacer actividad física" (Gotzone, 69).

"No había tiempo para el ocio, íbamos todos los días al campo a trabajar, a cuidar la tierra, el ganado" (Plácido, 79). 


\section{Edad adulta (20-59 años)}

Durante la edad adulta temprana (20-39 años), la falta de tiempo resulta tan evidente como en etapas anteriores, aunque por causas distintas, en función del género. El matrimonio, la crianza y otras responsabilidades familiares ocupan fundamentalmente a las mujeres. Para ellas, era habitual contraer matrimonio, tener hijos y abandonar sus trabajos fuera del hogar para cuidar de ellos. Entre los hombres, el trabajo es la causa principal de la falta de tiempo para la práctica físico-deportiva. El trabajo constituye el epicentro de sus estilos de vida, minimizando sus oportunidades para el ocio.

Los siguientes extractos reflejan esta hegemonía heteronormativa y la naturaleza diferencial de las barreras para la actividad físico-deportiva, según el género:

"A los 20 años me casé y no hice nada. Era una madre muy joven, cuidaba la casa, los niños, no tenía tiempo ¡Es diferente cuando te casas, cambias tu vida! Siempre me quedaba en casa." (Emilia, 74).

"En la edad adulta seguí trabajando y criando a mis hijos. Tuve cinco." (Flores, 76).

"Como adulto, tampoco hice actividad física. Siempre trabajando en el campo. No tenía opciones de ocio" (Manuel, 81).

Sin embargo, algunas personas pudieron practicar actividades físico-deportivas durante la edad adulta y, en muchos casos, continuar realizándolas durante largo tiempo:

"Después de los 20 años, disfrutaba el ciclismo y la lectura. El ciclismo, en grupo y en carreterra, hasta los 55 años. Casi 30 años andando en bici." (Germán, 70).

La edad adulta tardía (40-59 años) se muestra especialmente fértil para el inicio de la práctica físico-deportiva. De las 10 personas, nueve inician alguna actividad durante esta etapa o continúan practicando desde la adolescencia. Entre las mujeres, esto suele suceder en fases tardías de la edad adulta, cuando los hijos y hijas son más mayores e independientes.

"Comencé la actividad física hace unos 30 años, cuando mis hijos se fueron haciendo mayores" (Mercedes, 69).

Se confirma que de las personas del grupo físicamente activo (10) que practican actividades físico-deportivas en la edad adulta, sólo dos practicaban alguna en la adolescencia (Francisco, 64 años, fútbol y Natividad, 64 años, montañismo). Estas actividades $u$ otras parecidas son las que siguen practicando en la edad adulta. Sin embargo, entre las personas inactivas (ocho), son mayoría quienes no práctican ninguna actividad en la fase adulta (cinco de ocho). Únicamente Antonio (73) y Carmen (67) realizan alguna actividad durante su etapa adulta temprana.

Tercera Edad (60 años y más)

El análisis se centra ahora desde los 60 años hasta el momento del estudio. Para ello, se conjuga la mirada retrospectiva con la que se reconstruye la fase tardía de la vida físico-deportivo de este colectivo, con otra sincrónica, que permite la segmentación de la muestra en dos grupos, el de las personas físicamente activas (10) e inactivas (ocho). 
Se procede ahora a considerar las prácticas que realizan durante la tercera edad sendos grupos, si existe o no continuidad con prácticas anteriores, así como posibles factores implicados en la decisión de mantenerse físicamente activos o no en esta etapa.

Las prácticas más habituales son gimnasia colectiva, caminar, natación, actividades cardiovasculares y bailar. En muchos casos, estas prácticas son las mismas o están muy relacionadas con las iniciadas anteriormente. Solo una persona, Jose (74), se inicia en la tercera edad en el ocio físicamente activo, animado por su hija.

En el grupo de personas inactivas, se dan dos casos de abandono forzado de la práctica físico-deportiva por cuestiones de salud (Isabel, 67), Pilar (71). Ambas estaban físicamente activas antes de realizar la intervención quirúrgica y tienen intención de recuperar su práctica físico-deportiva, una vez tengan el alta médica.

"Ahora, no practico ninguna actividad debido a una operación de rodilla que tuve hace casi tres años. Pero a partir de ahora vuelvo a nadar. Había practicado natación antes (...) durante 20 años." (Isabel, 67).

Por su parte, Antonio permanece físicamente inactivo durante años por falta de motivación, tras experiencias no satisfactorias. Durante su estancia en una universidad inglesa, con algo más de 20 años, le obligaron a practicar a diario diversas actividades. Este carácter obligatorio hizo mella en su interés por la práctica físicodeportiva y permaneció inactivo durante el resto de su etapa adulta y tercera edad. Aún así, desea volver a practicar, pero siguiendo sus ritmos e intereses.

“Un día fútbol, otro rugby, tenis, natación... Los ingleses le daban mucha importancia al deporte. No me gustó, pero tenía que hacerlo. (...) incluso, cuando nevaba. Para mí fue un sacrificio." (Antonio, 73).

Estas tres personas, Isabel, Pilar y Antonio son las únicas del grupo físicamente inactivo que habían realizado actividades físico-deportivas en etapas previas o durante la tercera edad, como Pilar, desde su jubilación (natación).

La salud no siempre es causa de abandono. También impide el inicio de actividades. Es el caso de Mari Carmen (62) y Plácido (79). Ambos afirman que sus problemas de salud han sido la razón para no practicar:

“(...) Tengo migrañas y no puedo hacer esfuerzos, así que dejé de hacerlo." (Mari Carmen, 62).

"Tengo un problema con mi columna vertebral. Me gusta caminar, pero me cuesta mucho. Me canso y me duele ..." (Placido, 79).

Otro factor negativo para la adherencia físico-deportiva y que se pone nuevamente de relieve durante la tercera edad es el trabajo como valor primordial. Algunos testimonios de personas mayores inactivas evidencian esta centralidad y su impacto, mediante vidas dedicadas al trabajo, en las que no había tiempo para el ocio y actividades físico-deportivas.

"Mi vida siempre ha sido el trabajo". (Atiliano, 68).

"En la adolescencia no practiqué ninguna actividad físico-deportiva (...) De adulto, tampoco. Siempre he estado trabajando en el campo." (Manuel, 81) 
El caso de Flores es muy ilustrativo pues en el momento del estudio continúa trabajando, por voluntad propia, en el Hogar de Jubilados al que asiste:

"Cuando tenía unos 15 años, pude comenzar a trabajar en una casa. Luego, en una empresa, una tienda, un supermercado... Trabajando, siempre trabajando. (...). Ahora, estoy trabajando aquí (refiriéndose a la cafetería del Centro de Jubilados). Esto me lleva mucho tiempo." (Flores, 76).

Entre los factores que facilitan la adherencia destaca la existencia de instalaciones polideportivas públicas, cercanas a sus hogares o, en su defecto, una buena red de transporte público:

“No sé exactamente cuántos años llevo viniendo, pero creo que alrededor de 30 años. Está muy cerca de casa... (Gotzone, 69).

El apoyo familiar es clave para iniciar la práctica físico-deportiva en etapas tardías de la vida, cuando la capacidad de ser personas autónomas comienza a reducirse. El caso de José (74) ratifica esta cuestión. José no había realizado ninguna actividad físico-deportiva; sin embargo, animado por su hija, decide empezar en esta etapa a acudir a clases de ginmasia, impartidas en un polideportivo cercano de su casa:

"Para mí hacer actividad físico-deportiva es importante ahora. Hace un par de años, mi hija me dijo que, si no comenzaba a hacer alguna actividad físico-deportiva, dejaría de ser mi hija (risas)" (José, 74).

\section{Los itinerarios físico-deportivos}

Se trata ahora de reconstruir los itinerarios físicos-deportivos de las personas estudiadas y analizar si existen patrones distintos de evolución de la práctica y los factores implicados.

Para las personas físicamente activas, la edad adulta es un punto de inflexión para el inicio de la práctica físico-deportiva. Todas, excepto tres personas (Jose, 74, Francisco, 64 y Natividad, 64), inician alguna actividad en esta etapa y la mantienen hasta la tercera edad. José (74) es la única persona de este grupo que comienza a practicar en la última etapa, sin haber realizado ninguna actividad antes.

Así, considerando tres criterios, el inicio, la continuidad de las prácticas (más de cinco años de práctica) y si la persona está físicamente activa o no en el momento del estudio, emergen tres itinerarios entre las personas físicamente activas:

Itinerario 1. Activo precoz continuado. Aúna a quienes comienzan sus prácticas durante la adolescencia y continúan activos hasta el momento del estudio. Francisco (64) y Natividad (64).

Itinerario 2. Activo adulto continuado. Personas que se inician en este ámbito en la edad adulta y continúan hasta el momento del estudio. Gotzone (69), Esperanza (67), Emilia (74), Mercedes (69), Gabriel (68), Germán (70) e Inocencia (75).

Itinerario 3. Activo tardío. Quienes comienzan alguna actividad físico-deportiva a los 60 años o más y la continúan hasta el momento del estudio. José (74). 
Las trayectorias físico-deportivas de las personas inactivas revelan patrones distintos. De las ocho personas, solo tres tienen relación con este ámbito en etapas previas. Dos de ellas inician sus practicas durante su adolescencia (Isabel, 67 y Pilar, 71), pero tras trayectorias distintas, ambas acaban abandonando sus prácticas por motivos de salud en la tercera edad. Antonio (73) es el único que comienza a practicar durante la edad adulta temprana, abandona después debido a una mala experiencia con este ámbito. Los tres tienen intención de volver a la práctica, tras recibir el alta médica. Las cinco personas restantes no practican ninguna actividad físico-deportiva a lo largo de la vida.

En este escenario emergen tres nuevos itinerarios:

Itinerario 4. Activo precoz interrumpido. Comienzan en este ámbito durante la adolescencia, pero su actividad se interrumpe, estando inactivas durante el estudio. Isabel (67) y Pilar (71).

Itinerario 5. Activo adulto interrumpido. Se inician en la práctica físico-deportiva durante la edad adulta, pero abandonan sus actividades durante la tercera edad o antes. Antonio (73).

Itinerario 6. Inactivo permanente. Personas físicamente inactivas a lo largo de la vida. Flores (74), Plácido (79), Atiliano (68), Manuel (81) y Mari Carmen (62).

La Figura 1 representa los patrones de evolución de la práctica físico-deportiva de las personas mayores estudiadas a lo largo de sus vidas, identificando el tipo de itinerario al que se asocia cada uno.

Figura 1. Itinerarios físico-deportivos de las personas mayores estudiadas

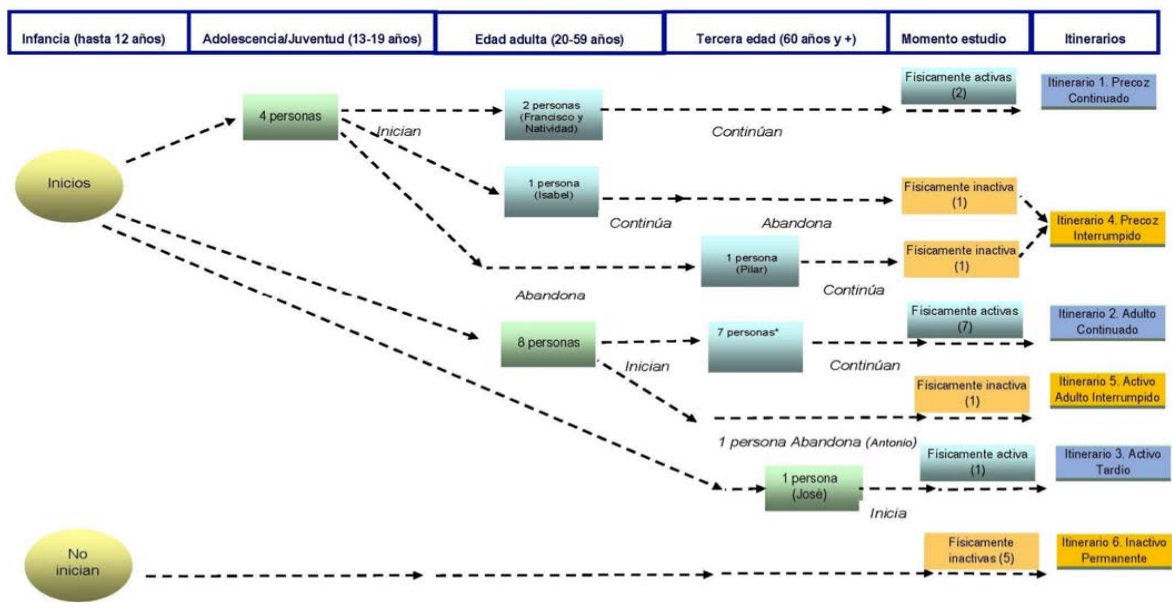




\section{Discusıón}

El objetivo del estudio era identificar los factores pasados y presentes que contribuyen o dificultan la práctica físico-deportiva durante la tercera edad. Para ello, se opta por una metodología cualitativa, mediante la que se reconstruyen y analizan los itinerarios físico-deportivos de este colectivo y su incidencia en su situación actual, como personas físicamente activas o inactivas.

Los hallazgos obtenidos no pueden ser interpretados al margen del contexto sociohistórico, en el que germinan las historias de vida analizadas. Un contexto de posguerra (Guerra Civil Española, 1936-1939) y una dictadura (1939-1975) que limitó las oportunidades para el ocio físico-deportivo de las personas estudiadas y modeló sus gustos, preferencias y estilos de ocio, especialmente durante su infancia y juventud, pero también en etapas posteriores.

Los seis itinerarios físico-deportivos identificados revelan que la relación de las personas de la muestra con la práctica físico-deportiva ha sido desigual entre ellas y en las distintas etapas vitales, pero en todos los casos, profundamente condicionada por las circunstancias del contexto y las oportunidades disponibles para aproximarse y disfrutar de este ámbito.

Las barreras estructurales, procedentes del contexto social, político, económico, etc. (Kleiber, McGuire, Aybar-Damali y Norman, 2008), poseen un claro protagonismo durante la infancia y adolescencia; primero, como falta de estímulos para la práctica físico-deportiva y escasez de iniciativas públicas estructuradas, inexistentes incluso en el contexto escolar; después, durante la adolescencia, una ocupación laboral precoz, ante la necesidad de contribuir a la economía familiar, incorporándose al mercado laboral o asumiendo tareas domésticas, cuidado de familiares o actividades agrícolas y ganaderas ${ }^{3}$. En ambos periodos, estas barreras les dificultaron el cultivo de sus intereses y aptitudes físico-deportivas, restringiendo su acceso a este tipo de experiencias y, en algunos casos, minando sus deseos de iniciarse y disfrutar de este ámbito, al estar inmersos en una cultura hegemónica, en la que el trabajo no era sólo un medio de vida, sino el valor supremo y principal fuente de sentido vital (ética del trabajo) (Elizalde, 2010).

Durante la edad adulta, las barreras más frecuentes, el trabajo, la crianza y las tareas domésticas, no dependen tanto del contexto, como del momento del ciclo vital y las circunstancias laborales de cada persona. Ahora bien, se trata de barreras personales (Kleiber et al., 2008), profundamente condicionadas por los estereotipos de género, de forma que el matrimonio, la maternidad y la carga de las tareas domésticas son, sobre todo durante la edad adulta temprana, los motivos principales de la falta de tiempo que impide o dificulta la práctica físico-deportiva entre las mujeres, mientras que el trabajo es la barrera primordial para los hombres. Esta distribución de funciones según género, sustento de las causas diferenciales de la no práctica físicodeportiva, era apenas cuestionada en un contexto de normalización social y plena aceptación de los roles que por género les correspondían (Rosón, 2016).

3. En la década de 1950 y 1960, el 40\% de la población activa en España se dedicaba a la agricultura $(39,8 \%)$, mientras que la industria representaba un $28,6 \%$ y los servicios un exiguo $27 \%$; Únicamente, el $27,7 \%$ de la población vivía en municipios de más de 100,000 habitantes (Tezanos, 2004), por lo que los estilos de vida eran profundamente rurales y marcados por importantes limitaciones económicas. 
Aún así, la edad adulta, en su conjunto y, especialmente para las mujeres durante la edad adulta tardía, constituye un tiempo de oportunidad para la práctica físico-deportiva, para continuar lo que ya practicaban en la adolescencia o explorar nuevas actividades que se mantienen en la tercera edad. El fenómeno del "fluorishing" es una realidad ya constatada en el ámbito del ocio, que permite el descubrimiento o recuperación de intereses y prácticas que por circunstancias diversas no pudieron ser realizadas en momentos anteriores (Cuenca, Kleiber, Monteagudo, Linde y Jaumot-Pascual, 2014). No se puede obviar, sin embargo, que, en este estudio, las prácticas realizadas están muy condicionadas por el género, siendo la gimnasia la actividad más habitual entre las mujeres y el fútbol o el ciclismo, entre los hombres. Se hace así patente el impacto de patrones heteronormativos, en los que el género determina, tanto los roles sociales que hombres y mujeres desempeñan, como el tipo de prácticas físico-deportivas que deben realizar (Arribas, Egido, Pastor y Aguado, 2009).

A la luz de los resultados, podría decirse que la edad adulta es un periodo estratégico para la vida físico-deportiva de las personas mayores. Prácticamente la totalidad de las personas físicamente inactivas en la tercera edad (seis de ocho), ya lo estaba en la edad adulta, debido a que la actividad físico-deportiva no les resultaba atractiva o no habían encontrado ni tiempo ni oportunidades para ello. Igualmente, casi todas las personas físicamente activas durante la tercera edad (nueve de 10) ya estaban activas en la edad adulta. Solo una persona (Jose, 74) se inicia en la práctica físico-deportiva en la etapa más tardía.

Las principales barreras durante la tercera edad y, que afectan, sobre todo, a las personas inactivas, son personales: salud, como motivo de no práctica o abandono y experiencias físico-deportivas previas negativas, que reducen la motivación. En esta fase, se hace patente, además, la importancia de barreras ya existentes, como la ética del trabajo, cuyo impacto persiste en la tercera edad, cuando se dispone de más tiempo libre y oportunidades para la práctica físico-deportiva. El caso de Flores (74), con una vida dedicada al trabajo, permite entender que la hegemonía de esta ética del trabajo no afecta únicamente a los hombres y que una de sus consecuencias es la falta de interés en este ámbito, hasta el punto de que, tras la jubilación, se prefiere continuar trabajando, que explorar las posibilidades que el ocio y el ámbito físico-deportivo ofrece. Un hecho que reafirma, en primer lugar, la naturaleza compleja y dinámica de las barreras, en cuanto que una barrera estructural (ética del trabajo, falta de oportunidades, ausencia de iniciativas organizadas) puede tornarse personal, al ser percibida y asumida como una seña de identidad (falta de interés en la práctica físico-deportiva); y en segundo lugar, la temporalidad del impacto de las barreras, cuyo efectos pueden hacerse patentes de manera inmediata o a medio o largo plazo, en fases posteriores al momento en el que la barrera se activa.

Aunque su protagonismo es menor que el de las barreras, algunos factores facilitadores de la práctica físico-deportiva han sido esenciales para la promoción de la vida físico-deportiva de las personas estudiadas en las distintas etapas de la vida: la orientación natural hacia el juego activo y el movimiento, durante la infancia; el abandono de tareas asociadas al cuidado de los hijos e hijas, para muchas mujeres adultas; la proximidad de los centros polideportivos locales y el apoyo familiar son los factores facilitadores que más contribuyen a la adhesión de las personas mayores a la actividad físico-deportiva. 
La tabla 2 resume los factores determinantes de la práctica físico-deportiva de las personas estudiadas en cada fase vital.

Tabla 2. Factores determinantes de la práctica físico-deportiva a lo largo de la vida

\begin{tabular}{|l|l|l|l|l|}
\hline \multicolumn{1}{|c|}{ Factores } & \multicolumn{1}{|c|}{$\begin{array}{c}\text { Infancia } \\
\text { (hasta 12 años) }\end{array}$} & $\begin{array}{c}\text { Adolescencia y } \\
\text { Juventud (13-19 } \\
\text { años) }\end{array}$ & $\begin{array}{l}\text { Edad Adulta (20- } \\
\mathbf{5 9} \text { años }\end{array}$ & $\begin{array}{l}\text { Tercera Edad (60 } \\
\text { años y +) }\end{array}$ \\
\hline Facilitadores & $\begin{array}{l}\text {-Juego espontáneo, } \\
\text { liderado por el } \\
\text { movimiento. }\end{array}$ & - Ninguno. & $\begin{array}{l}\text { - Más tiempo } \\
\text { libre, cuando } \\
\text { hijas e hijos son } \\
\text { independientes, } \\
\text { entre las } \\
\text { mujeres. }\end{array}$ & $\begin{array}{l}\text { - Proximidad } \\
\text { de centros } \\
\text { polideportivos. } \\
\text { - Apoyo familiar. }\end{array}$ \\
\hline Barreras & $\begin{array}{l}\text { - Ausencia de } \\
\text { iniciativas públicas } \\
\text { estructuradas y del } \\
\text { contexto escolar. }\end{array}$ & $\begin{array}{l}\text { - Colaboración en } \\
\text { responsabilidades } \\
\text { familiares y } \\
\text { laborales. }\end{array}$ & $\begin{array}{l}\text { - Trabajo, } \\
\text { crianza y tareas } \\
\text { domésticas. } \\
\text {-Estereotipos } \\
\text { de género, en } \\
\text { la elección de } \\
\text { actividades. } \\
\text { - Ética del } \\
\text { trabajo, propicia } \\
\text { falta de interés. }\end{array}$ & $\begin{array}{l}\text { - Ética del } \\
\text { trabajo, propicia } \\
\text { falta de interés. } \\
\text { - Experiencias } \\
\text { previas } \\
\text { negativas. }\end{array}$ \\
& & & &
\end{tabular}

\section{Conclusiones}

Este estudio confirma que la clasificación de las personas mayores en dos grupos, físicamente activas e inactivas, resulta insuficiente porque ignora cuestiones, como un pasado físicamente activo o la intención de retomar la práctica, tras un periodo de abandono forzado de la actividad. Aspectos esenciales a la hora de explicar las decisiones de este colectivo de estar o mantenerse físicamente activo durante la tercera edad. Los seis itinerarios identificados delinean con precisión los procesos y factores implicados en el ocio físico-deportivo actual de las personas mayores y apuntan la necesidad de avanzar en el diseño efectivo de estrategias longitudinales de intervención, orientadas a favorecer a adhesión a este ámbito a lo largo de la vida.

La mirada retrospectiva y longitudinal, inherente al concepto de itinerario, permite ratificar que, además de los factores determinantes (facilitadores y barreras) implicados en "el aquí y el ahora" de las personas mayores, cuyo impacto es clave en su decisión de estar físicamente activas o no en la tercera edad (salud, apoyo familiar y proximidad de centros polideportivos), se deben contemplar otros, asociados a la historia de vida de este colectivo (importancia del juego y movimiento en la infancia, falta de oportunidades, iniciativas organizadas o formación en Educación Física, experiencias previas con la práctica físico-deportiva, etc.) y cuyos impactos no han de ser necesariamente inmediatos o puntuales, pudiendo afectar a las relaciones con este ámbito a lo largo de toda la vida. Su papel es clave para la configuración de los 
itinerarios físico-deportivos de las personas mayores y también para entender su estilo de ocio actual. De ello se deriva que el propio itinerario físico-deportivo constituye un factor determinante, por su capacidad de favorecer la práctica físico-deportiva (itinerario 1,2 y 3 ) o dificultara a lo largo de la vida, incluida la tercera edad (itinerario 4, 5 y 6). Así, haber practicado alguna actividad antes de la tercera edad, si la experiencia fue positiva, puede favorecer la adherencia físico-deportiva en etapas tardías de la vida. Por el contrario, no haber practicado ninguna actividad o haber vivido experiencias negativas, puede dificultar la práctica físico-deportiva en esta etapa, minando el interés por estas actividades.

La edad adulta emerge en esta investigación como un periodo óptimo para iniciarse, cuando previamente no se han dado las condiciones para ello. Aún más, la importancia de este periodo radica en la tendencia identificada a continuar durante la tercera edad, tan alejado o cercano a la práctica físico-deportiva como se esté en la edad adulta. Una tendencia que, por supuesto, no es ajena al impacto de factores determinantes que puede provocar el abandono forzado de este ámbito, pero que evidencia una "intención de continuidad" en los estilos de ocio físico-deportivo de las personas mayores que, de confirmarse, podría ser muy relevante en el diseño de estrategias de intervención a favor de la adherencia físico-deportiva en la tercera edad.

El contexto sociohistórico, con sus circunstancias culturales, económicas, políticas, educativas y familiares, resulta ser un factor determinante de la práctica físicodeportiva, pues se ha demostrado su influencia en la configuración de los itinerarios físico-deportivos de las personas mayores. Elementos generales del contexto, compartidos por personas de una o varias generaciones, imprimen su impronta, no solo en términos de derecho al ocio físico-deportivo (oportunidades reales para su disfrute), sino también en clave de valores, asociados a la forma de entender, disfrutar y cultivar este ámbito. Las historias analizadas poseen un componente personal innegable, responsable de la singularidad de cada itinerario, pero también un componente social común, en cuanto narraciones gestadas en la interacción con otras personas y en un contexto concreto (Sparker y Devis, 2008). Por eso, estudiar los itinerarios de ocio físico-deportivo permite comprender, tanto las historias de vida, como el contexto social que las acoge (Monteagudo, 2019). Se evidencia así que la subjetividad entraña siempre una dimensión social.

A pesar de sus limitaciones (muestra intencional, vinculada a un contexto socio-histórico concreto, de ámbito exclusivamente urbano), este estudio ha demostrado que la apuesta por la adherencia físico-deportiva de las personas mayores exige intervenir, no sólo en la tercera edad, sino también en etapas anteriores, propiciando las condiciones idóneas para la instauración del hábito físico-deportivo a lo largo de la vida. Estrategia ineludible si se quiere hacer de este ámbito, una oportunidad real para la mejora de la salud y bienestar de este colectivo.

\section{Agradecimientos}

La Fundação de Amparo a Pesquisa do Estado de Minas Gerais (FAPEMIG), por el financiamento de la beca de estudio posdoctorales de uno de los autores. 


\section{REFERENCIAS BIBLIOGRÁFICAS}

Aitchison, C. C. (2003). Gender and leisure. London: Routledge.

Aparicio, V.A., Cabonell, A. y Delgado, M. (2010). Beneficios de la actividad física en personas mayores. Revista Internacional de Medicina y Ciencias de la Actividad Física y el Deporte, 10(40), 556-576.

Arribas, J. C. M., Egido, L. T., Pastor, V. L. y Aguado, R. M. (2009). Factores que determinaron una educación física y deportiva de género durante el franquismo. Apunts Educación Física y Deportes, 98, 5-14.

Ayuntamiento de Bilbao (s.f.). II. Plan. Bilbao Ciudad Amigable con las Personas Mayores 2016-2019. Área de Acción Social. Consultado el 20 de diciembre 2018. http://www.mayoresdebilbao.com/wp-content/themes/B60/pdf/II_Plan_ Amigable_CAST.pdf

Brown, P. R., Brown, W. J., Miller, Y. D. y Hansen, V. (2001). Perceived constraints and social support for active leisure among mothers with young children. Leisure Sciences, 23, 131-144.

Clark, V. L. P. y Creswell, J. W. (2008). The mixed methods reader. London: Sage.

Castañeda Vázquez, C., Campos Mesa, M. D. C. y Garrido Guzmán, M. E. (2011). Personas mayores: dificultades hacia la práctica deportiva y posibles causas de abandono. Trances: Revista de Transmisión del Conocimiento Educativo y de la Salud, 4(3), 511-526.

Cuenca, J., Kleiber, D. A., Monteagudo, M. J., Linde, B. D. y Jaumot-Pascual, N. (2014). The influence of meaningful leisure on the subjective well-being of older adults in the Basque Country of Northern Spain. World Leisure Journal, 56, 120129. https://doi.org/10.1080/16078055.2014.903721

Currie, J. (2004). Motherhood, stress and the exercise experience: Freedom or constraint? Leisure Studies, 23, 225-242.

Elizalde, R. (2010). Resignificación del ocio: Aportes para un aprendizaje transformacional. Revista Polis, 25, 437-460.

Eustat. Instituto Vasco de Estadística (2019). Censo población de Euskadi. https:// www.eustat.eus/municipal/datos_estadisticos/bilbao_c.html.

Guthold, R., Stevens, G. A., Riley, L. M. y Bull, F. C. (2018). Worldwide trends in insufficient physical activity from 2001 to 2016: a pooled analysis of 358 population-based surveys with 1.9 million participants. Lancet Glob Health, 6, 10771086. https://doi.org/10.1016/S2214-109X(18)30357-7

IMSERSO (2017). Informe 2016. Las personas mayores en España. Madrid: Ministerio de Sanidad, Servicios Sociales e Igualdad.

Kleiber, D., McGuire, F. A., Aybar-Damali, B. y Norman, W. (2008). Having more by doing less: The paradox of leisure constraints in later life. Journal of Leisure Research, 40(3), 343-359. https://doi.org/10.1080/00222216.2008.11950144

Lakatos, E. M. y Marconi, M. (2003). Fundamentos de metodología científica. 5. ed. São Paulo: Atlas.

Langley, D. J. y Knight, S. M. (1999). Continuity in sport participation as an adaptive strategy in the aging process: A lifespan narrative. Journal of Aging and Physical Activity, 7(1), 32-54. 
Lopes, J. A., Longo, G. Z., Peres, K. G., Boing, A. F. y Arruda, M. P. D. (2010). Factors associated with insufficient physical activity: a population-based study in southern Brazil. Revista Brasileira de Epidemiologia, 13(4), 689-698. https://doi. org/10.1590/S1415-790X2010000400013

Martín, M., Moscoso, D., Martínez del Castillo, J. y Ferro, S. (2009). La práctica deportiva en España: Desigualdades entre mujeres y hombres en la población joven, adulta y mayor. Actas I Congreso Internacional de Cultura y Género: La Cultura en el Cuerpo. Elche: Universidad Miguel Hernández

Masnou, M. y Puig, N. (1995). El acceso al deporte: Ios itinerarios deportivos. En D. Blázquez (Ed.), La iniciación deportiva y el deporte escolar (pp. 371-394). Barcelona: Inde.

Meihy, J. C. S. B. (2006). Os novos rumos da história oral: o caso brasileiro. Revista de história, 155, 191-203.

Monteagudo, M. J. (2019). El ocio de las personas mayores en España. Avances en el conocimiento de sus experiencias e itinerarios desde la investigación narrativa. En A. B. Rodríguez., H. Ferreira y M. M. Baptista, Ocio y cambio social. Oportunidades y desafios para el desarrollo humano en Iberoamérica (pp. 23-48). Bogotá: Kinesis.

Monteagudo, M. J. y Cuenca, M. (2012). Los itinerarios de ocio desde la investigación: tendencias, retos y aportaciones. Pedagogía social. Revista interuniversitaria, 20, 103-135.

Nahas, M. V.; Goldfine, B. y Collins, M. A. (2003). Determinants of physical activity in adolescents and young adults: the basis for high school and college physical education to promote active lifestyles. Physical Educator, 60(1), 42-56.

Pérez Díaz, J., Abellán García, A., Aceituno Nieto, P. y Ramiro Fariñas, D. (2020). Un perfil de las personas mayores en España. Indicadores estadísticos básicos. Madrid: Informes. Envejecimiento en Red. CSIC.

Picorelli, A. M. A., Felicio, D. C., Pereira, D. S., Pereira, L. S. M. y Sherrington, C. (2014). Adherence to exercise programs for older people is influenced by program characteristics and personal factors: a systematic review. Journal of Physiotherapy, 60, 151-156. https://doi.org/10.1016/j.jphys.2014.06.012

Rosón, M. (2016). Género, memoria y cultura visual en el primer franquismo. Madrid: Cátedra. https://doi.org/10.7203/KAM.12.11136

Sparkes, A.C. y Devís-Devís, J. (2008). Investigación narrativa y sus formas de análisis: una visión desde la educación física y el deporte. En W. Moreno (Ed.), Educación cuerpo y ciudad: El cuerpo en las interacciones e instituciones sociales (pp. 43-68). Medellin: Funámbulos Editores.

Tezanos, J. F (2004). España: Estructura y dinámica social. Logroño: Instituto de Estudios Riojanos.

Weinberg, R. S. y Gould, D. (2014). Fundamentos da Psicologia do Esporte e do Exercício. Champaign: Human Kinetics.

WHO. World Health Organization. (2004). Envelhecimento ativo: uma política de saúde. Brasilia: Organização Pan-Americana da Saúde.

WHO. World Health Organization. (2015). World report on ageing and health. https://www.who.int/ageing/publications/world-report-2015/en/ 